\title{
TREAT TO TARGET IS NOT LINKED TO BEST MYOCARDIAL STRAIN INDICES: IS THERE REALLY A MYOCARDIAL DYSFUNCTION DIRECTLY ASSOCIATED WITH DISEASE ACTIVITY?
}

Alisson Aliel Vigano Pugliesi (Universidade Estadual de Campinas, Campinas, SP, Brasil), Hugo Vahid Rahnemaye Rabbani (Universidade Estadual de Campinas, Campinas, SP, Brasil), José Roberto Matos Souza (Universidade Estadual de Campinas, Campinas, SP, Brasil), Manoel Barros Bertolo (Universidade Estadual de Campinas, Campinas, SP, Brasil)

\section{BACKGROUND}

The risk of developing heart failure (HF) is twice as high in the RA population. It is hypothesized that, in addition to the atherosclerotic ischemic component and the traditional risk factors, there is a mechanism of cardiotoxicity related to the inflammatory pathways. A few previous studies indicate an association between disease activity and myocardial dysfunction, but they involved elderly population with comorbidities and in use of TNF- $\alpha$ inhibitors. Our main objective is to evaluate the correlation between clinical disease activity index (CDAI) and global longitudinal strain (GLS) - the most sensitive echocardiographic method to detect changes in ventricular contractility - in a RA population without cardiovascular risk factors and using only conventional synthetic DMARDs.

\section{MATERIALS AND METHODS}

From June 2016 to January 2019, RA patients were selected to perform transthoracic speckle tracking echocardiography for GLS evaluation. Patients aged 18-60 years and classified according to ACR 2010 criteria were included. Patients with diabetes mellitus, uncontrolled hypertension, smokers, BMI>35, atherosclerotic disease history, and in use of biological medication were excluded. Patients underwent a clinical evaluation in which a single physician measured the CDAI and, within a period of one month, an echocardiographer blinded to the patient's clinical data assessed the GLS. Student's t-test and Spearman correlation were used for statistical evaluation.

\section{RESULTS}

Thirty-six patients (91.7\% female, with ages ranging from 16 to 56 years) participated in the study. No correlation was found between CDAI and GLS values (Graphic 1). There was no adequate accuracy between values of CDAI $\leq 10$ and $\mathrm{GLS}<-18$ and $\mathrm{GLS}<-20$ (values considered normal), according to Table 1. The presence of RF, anti-CCP and the disease duration were also not associated with CDAl values.

\section{CONCLUSION}

Data from this study (small but with highly selected population) do not corroborate the association of CDAl with myocardial dysfunction and question whether there is a cardiotoxicity factor linked to the disease. Traditional risk factors, subclinical atherosclerotic disease, and cardiotoxic drug effects should be the subject of studies designed to explain the higher prevalence of $\mathrm{HF}$ in the RA population. 